\title{
Distributed Leadership Theory in Creating Capabilities and Learning Outcomes in Higher Education: An Analysis of Online Leadership
}

by Sarah-Taylor Gough ${ }^{1}$

iHeartTeaching, USA

\begin{abstract}
Online leadership of higher education institutions (HEIs) is conducted on Twitter. By highlighting the existing patterns of interactions, distributed leadership (DL) is not only eminent in its simplest form, but collegiality and autonomy can readily be realized. New knowledge, based on the tweets and collegial online interactions from the 14,183 tweets sent by the HEIs in the US, UK, Canada, and South Korea, not only promotes the HEIs' unique online persona, but captures the very essence of leadership under investigation. Distinct leadership styles are separated into any one of the twenty-one administrative activities, which I constructed. Further, the learning opportunities and outcomes, associated with either managerial and non-managerial functions, culminate as the distinguishing features of online leadership. Contextual analysis has been applied to navigate the open conversations and the interactions taking place to categorize and measure the impact of the leadership in the ongoing practice of DL as a demonstrative theory. Long-term instruction in all its administrative roles is favored as the means for providing a new form of education - tweet by tweet. How the learning objectives are being advanced in the tweets themselves governs not only the resulting leadership style, but predicts the learning process in HE.
\end{abstract}

Keywords: distributed leadership, higher education, online leadership, administration, social media

* Corresponding author at: iHeartTeaching, NY, USA.
E-mail address: research@iheartteaching.org
Authored date: November 11, 2017

Copyright (C) iHeartTeaching 2017, All rights reserved

\section{I- INTRODUCTION}

\section{Background}

Higher education (HE) institutions in the past decades have coped with financial, competitive, demographic, internationalization and standardization hurdles by streamlining the management of teaching and learning into a structured industry laden with contemporary shortcomings (Stensaker and 
Norgård, 2001). Ushering the advent of 'New Public Management' (Floyd and Preston, 2017; van Ameijde et al. 2009), this paradigm marks the transition from an independent, traditional education structure to one that is common with the adaptation of industrial and market forces. Also deemed as 'New Managerism' (Blaschke et al. 2014), inevitable challenges arose. Consequently, HE institutions adopted managerial ideals, which had been jettisoned by the private business sector, and thus rendering collegial values of university leadership to that of a culturally unfit loss. Distributed leadership (DL) would be the ideal contender amid the need for remedies (van Ameijde et al., 2009). DL theory posits that individuals across different departments and levels are engaged within shared decision-making situations that are less hierarchical. Presumably, a practical demonstration of DL in action can be attributed to the use of social media, such as Twitter, by HE institutions to promulgate succinct messages to students, faculty members, and the general public (Veletsianos et al. 2017). This practice is growing with importance, specifically in the HE sector through the use of new social network platforms (Peacock et al. 2016).

\section{Rationale of the Framework for the Study}

To frame the importance of the benefits of DL, contextual analysis has been employed to make meaning out of the communications syndicated on Twitter. It becomes evident that all participants involved are no longer limited to being followers, as the 'followership' is permitted as equals in the spirit of DL theory, enabling the online leadership of the institution to manifest itself in functions which

are two-fold. By separating the online leadership functions via DL as either being non-managerial or managerial, which are distinct roles. Conducive to DL theory, not only are the administrative roles maintaining collegial values and promoting leadership akin to the institution's mission and vision, the 
university culture and its leadership can promote competitive advantage without invoking the flaws of new managerism. At this point, the act of tweeting fits the operational meaning of leadership. The tweet that is sent from an HE institution constitutes authority with a leading voice that should not be confused strictly with the limiting role of public relations. Each tweet plays the role of contributing towards the cultural dynamics of leadership values. Referring to the above-mentioned DL taking place across the distinct operations, tweeting legitimizes the following conceptual properties within HE networks, which substantiate leadership in its precise categories. The quality of teaching and learning can only be enhanced in the learning opportunities and outcomes by opening DL to an unlimited number of online participants. The content of the Twitter messages are sorted into twenty-one online leadership activities to explore how each of the selected HE institutions perform distributed instruction. Each online leadership activity is enacted as either an opportunity or an expected end to present its role (Gough, 2017b). If the activity contextualized in the public post is clearly an opportunity as a means, then its enactment towards improving teaching and learning is a capability. Such a capability happens to be a non-managerial leadership role. Otherwise, if the enactment is an expectation of end, then the managerial activity (function) is outcome-based towards learning. Thus online leadership is configured into twelve possible capability-based, non-managerial activities and nine possible outcome-based, managerial functions, which I have constructed.

\section{The Purpose of the Study}

The apparent leadership approach is executed on a day-to-day exchange as the Twitter communications being boldly practiced by HE institutions in the four regions, US, England, Canada, and South Korea, will be pinpointed, sorted, and tallied into relative frequencies to determine the 
leadership trajectories that support its dynamic role in the distributed online pipeline. The purpose of this research is to explore the leadership practice of HE on Twitter in order to provide explanations of their online leadership performance that can be observed as being an accumulation of the twenty-one online leadership activities. The assumption made in this study is that although there will be differences between Western and South Korean HE institutions in terms of management culture and operations, the individual HE institution's organizational culture in relation to Twitter usage ascertains the leading trends to adopt technology as a way to reach its desired network's leadership outcomes. As such, it would be meaningful to trace and summarize the administrative and leadership roles of universities across different continents and cultures.

\section{Research Questions}

This research questions stated below will contribute to the growing number of empirical studies on HE that run DL as its modus operandi. Practitioners of DL have independently justified their attempts to broaching DL as organizational models to study new forms in decision-making, collaboration, and team settings. The implications are inchoate, whether theoretical, conceptual, or experimental (van Ameijde et al. 2009). Fortunately, this study's investigation into online leadership of HE is favorable both empirically and structurally as an 'experimental' and normative remedy, respectively, to the theory that shall reinforce its conceptual undertaking.

The following research questions for this study are:

RQ1: What are the quantifiable interpretations from the emotional responses given to the online 
administrative activities, initiating interaction for each education department or university?

RQ2: What are the quantifiable interpretations from the nine defined managerial functions which are outcome-based online administrative activities for each education department or university?

RQ3: What are the quantifiable interpretations from the twelve defined non-managerial opportunities which are capability-based online administrative activities for each education department or university?

\section{II- LITERATURE REVIEW}

\section{Distributed Leadership}

DL theory is about decentralizing the authority given to decision-making from a sole leader to multiple individuals across various departments and levels in the organization. DL theory persuades traditional hierarchies to favor a less hierarchical and a more shared decision-making approach (Hargreaves, 2007; Spillane, 2006). It focuses on the concept of interdependency between multiple leaders and its subordinates, rather than the common situation of non-official members being dependent on the commands and instructions from their designated leaders (Spillane et al. 2007b), which commonly occurs in decentralized and dispersed leadership structures.

This DL theory promotes contexts in which individuals are given the opportunities to lead with expertise. The principal of the school or president of the organization, such as a city college or a worldclass university, and its senior leaders may adopt a strategic approach in how they develop professional 
opportunities in the form of training and guidance to junior officers and non-managerial members, who have been assigned to lead based on their level of expertise. DL theory is significant in the leadership context because it provides the culture to facilitate effective school improvement (Gunter et al. 2013; Goldstein 2004; Gronn 2008; Harris 2004, 2005; Harris and Spillane, 2008; Leithwood et al. 2009; Spillane 2006; Spillane et al. 2008), whereas decentralized and dispersed leadership theories lack this kind of function.

DL theory lacks the diagnosis of situations and feelings that are channeled by online leaders in network settings. Also, without structure for the DL theory to posit on social media communications, there is no way to analyze how messages that are disseminated in such online platforms can be restructured to achieve its priorities in the management pipeline. That stated, this research is significant, as well as for being the first to analyze in a simple form how DL theory can be extended to online leadership in social media. Applying DL theory to the usage of the online platforms will be auspicious to understand the current impact of the Fourth Industrial Revolution, as social media has a far greater influence on the average person today. Spillane et al's (2004) expressed that the distributed perspective of being "multiplicative rather than [an] additive model" holds true in the case of social media, simply “... because the interactions among two or more leaders in carrying out a particular task may amount to more than the sum of those leaders' practice" (p. 16). In the case of online leadership conducted by HE via Twitter, this distributed perspective provides an opportunity to link the impact of a single, online Tweet upon the numbers in its connected readership, known as followers. This distributed perspective encourages both the practitioner and researcher to go beyond the physical organizational structure's constraints and boundaries in the traditional, governance-based, collegial leadership style (Blaschke et al. 2014; Floyd and Preston, 2017; Stensaker and Norgård, 2001; van Ameijde et al. 2009) and 
converge with the online tapestry of Twitter built on the ideas of new public management, or with respect to new managerism.

\section{Leadership and Online leadership}

The online distribution of knowledge and activities foregrounds the leadership's capacity to state its tasks by transferring the information directly to its network of members. Openness and candidness in the digital connectivity permit any single leader or follower to not only acquire new forms of cognition, but also to diffuse knowledge and emotions with new ways of thinking to the extent that the individual agency by a single actor can be influential as much as a collective action would. In such an organizational design of which the managers monitor, observe, and assess what each participant says or does, the many instantaneous interactions add to any given routine, inquiry, tool, or situated process, as conversations bring insights towards better understanding and decision-making. Unlike traditional, dynamic and practical DL approaches, online leadership hosts an analytical perspective on the organizational activities being enacted for all participants of the online network to influence the above-mentioned communications into manageable units, such as situational opportunities and functional outcomes.

One may ask, how does Twitter-centered online leadership contribute to HE for the practitioner and researcher? For starters, online leadership, in this particular study can be viewed to consummate DL theory insofar as a digital practice that is the product of all interactions by its connected parties on a social media platform or the Internet, whereby the activity in its entirety is stretched out and distributed electronically across any human, machine, or thing. More important, online leadership in HE is the contemporary approach taken by university departments and colleges to distribute both opportunity- 
and outcome-based administrative activities across its faculty, students, and the general public to spur academic interests, knowledge, school spirit, and thinking into active learning, academic life and university affairs, societal challenges, and professional development (Gough, 2017b).

Academic proponents to South Korea's adoption of the Fourth Industrial Revolution have already adeptly coined the current government administration's aims, efforts, and challenges into fostering social media and other interactive communication channels for its constituents as "Presidential Leadership" (Eom, 2017). No other school of thought besides what is emanating from Seoul National University's public administration department has been explicit on the promotion of digital leadership, determined by presidential leanings. Being inspired by such an undertaking to open a long-awaited perspective on the realized trends and its many anomalies, "online leadership" is therefore not only semantically appropriate, but describes the obvious policy to engender innovative solutions by any organizational administration. Reverting to Eom (2017) that more or less addresses online leadership under the auspices of the current presidential administration of President Moon Jae-in, two concepts have been distinguished as: firstly, leadership in the digital age, encompassing both operational leadership and structural leadership, as well as elements of awareness building, resource mobilization, nurturing a specific type of attitudes and skills, knowledge, and experiences; and secondly, as digital leadership, for having a quality of "organizing without organization" and both responsive and responsible leadership styles defining the cyber-physical system (CPS) and similar systems in which all stakeholders collaborate within a broad network, rather than isolating technologies as a separate system in itself, to explain for the paradigm shift in leadership.

This kind of online DL as a means of appropriating knowledge is contributing immensely to the existing literature on DL theory. The online DL theory I propose in practice can surely integrate with 
knowledge work van Ameijde et al (2009) referred to as a means to spur resiliency in the face of an organizational transition phase from the traditional academic-based and collegial governance styles to a new managerism or new public management purpose. Blaschke et al (2004) describe the "peripheral issues [of leadership, governance, and management which] offer additional services to the public: knowledge and technology transfer (KT) and academic education and lifelong learning (AL)" (p.6). Floyd and Preston (2017) review middle leadership roles, namely, Associate Deans in UK universities in terms of how they perform both their management and budgetary responsibilities, while working in a "fast-changing and increasingly complex environment" involving more adaptation of DL models in the work process consisting of more "cross-institutional project work and initiatives" (p.941). Floyd and Preston (2017) explain "theoretically at least, to a genuinely distributed leadership model and allow for institutions to react with more speed and flexibility, and be more innovative and entrepreneurial in their approach to teaching, research, and knowledge transfer" (p.941). This study significantly contributes by proposing the concept of online DL as an added layer to the previous literature on DL theory. The insights on online DL is current, liberating and profound, while making sense of the communication and knowledge being available to everyone, and capturing and quantifying online DL trends of selected universities in four nations, namely, UK, Canada, USA, and South Korea.

\section{Distributed Leadership in Practice}

It is common for HE in the US and Canada to utilize social media functions as a daily routine to focus on maintaining a positive rapport with students. As such online activities persist to constitute a lion's share of administrative roles and functions, the students, administrators and faculty members, who are the ultimate main end-users, may very well interact by sharing both feelings and ideas. By 
reflecting on all learners' interests, pursuits, professionalism, and changes to those desires, the network of users thus influences the leadership at the helm. Further, the task for North American university administration and faculties has been a forward-looking challenge to reorganize their administrative routines such that a given activity produced in the online pipeline via social media not only distribute cognition, but also may purvey an understanding of feelings, often directed both to and fro with its constituents of influence. Leaders steer towards the changes in the demands by students and its faculty members as activities for the moment are planned in advance to constitute the emergent properties of communicating both opportunities and outcomes for learning and the management of its clients and youth. In particular, Canadian HE and its academic institutions have succeeded in constructing its own narratives that resonate with the student body and staff. South Koreans, on the other hand, often use a smartphone-based social networking and chatting platform, called "KakaoTalk," for organizing all contacts into manageable private group settings (Gough, 2017a).

Moving forward, Spillane et al. (2004) defines the constituting leadership activity in the DL theory as memos "represent[ing] a crucial tool that contributes to defining leadership practice" (p. 25). Education departments are voicing their activities with Twitter accounts akin to the way previous schools had done in Spillane's proposed use of memos. In the same vain, Tweets can be thought as short e-memos for both formal and informal notices. This perspective can facilitate the practice of DL for both the practitioner and researcher when they view tweets as e-memos. In this regard, the online leadership framework is first to claim that tweets can be viewed as e-memos, conceptually streamlined as leadership-driven tools to authorize, command, collaborate, express, elaborate, and publish. The capabilities thus being mediated by its leaders to the others imply that leaders are addressing its recipient, who takes on the role of a follower or observer. Even better, this theoretical scope of e- 
memos treats individuals as always alternating between the role of either being a leader, passive observer, or an active follower. This distinction clearly allows for dichotomy to take place in any organizational structure. Since individuals are capable of sending e-memos as a leader would, and technically allow others at the other end of communications to follow and observe, as any good listening, guessing, reading, or observing leader should, there is a dynamic nature of alternating roles that represents human nature in power balances. This alternation of sending, receiving, as well as actively following tweets is what maintains the exchanges of leadership with fluidity. Knowledge is carried forward in the distribution grid, as one leader would to another, and simultaneous communication with dynamic memos, tracing the transformation of the entire organization's trajectory of leadership with core organizational values. The model of tweets rests on the contributions of individual e-memo holders. Further, the e-memos support the concept of blending both physical and virtual realities in a means to holistically perform genuine DL online. Meeting agendas serve as a highly structured, event-based artifact that is influential in terms of guiding the conversations in meetings and on informing the community (Spillane et al. 2004). The role of the agendas varies both within and across a leadership activity (Spillane et al. 2004), and this resonates throughout the statistical findings, shown in the frequency tables in the Appendix. Likewise, electronic agendas (eagendas) for meetings and real-time updates that turn to specific events and news can also be spread across its community-at-large-directly online. The social media platform, then, bodes well as a tool that is open to the possibilities in collaborating and promoting a sense of shared responsibility with its followers. This study is the first to add value to DL in the context of agendas by extending it to eagendas being used online to invoke DL.

Moreover, the positioning of online activity that is constitutive in the contemporary HE context 
is rather conventional in the UK's practice of DL. Although the UK has increasingly applied DL theory to restructure the school organization over the past decade, online leadership has shown moderate progress. South Korea's top universities, as shown in the analysis, have only begun to recognize the long-term benefits of constituting an online presence for administrative activities, as more capabilities and outcomes being distributed in the past year are also constituting the narratives that the traditional leadership wishes to seek.

\section{Twitter as a Data Source}

According to Twitter (2017), there are over 100 million Twitter users daily, 67 million Americans users, and 13 million UK users. It is estimated that about $80 \%$ of the Twitter accounts are managed outside of the US. While education departments at universities promote information and news to their students, as well as broader audiences, Twitter usage can convey the cultural dynamics for how leadership, interpersonal and cultural communication styles converge on an interactive setting for its users, endemic to US, UK, and Canada. The study sets out to capture that exact essence, online. Since Twitter has a limit of 140 characters in length for brevity, it is convenient for researchers to understand the context in a straightforward, clear, and flexible manner. In addition, more interactive features add value to the emotional experience: analysis may now go beyond parsing of text, including hashtags (\#) and mentions (@) by jointly discerning the value attributed to linked photos and videos. Such an advantage for enriching engagement with a wider audience is intended for review in this study.

In previous studies regarding the professionals' usage of Twitter in the education setting, 15 days of Twitter activities were analyzed to see how the Twitter platform was used by 17 American and 
Canadian school administrators for professional learning (Cho, 2016). A single week of Twitter interaction corresponding to a direct research question, "Why do you use Twitter?" occurred in Twitter involving participants in Turkey to distinguish their user behavior (Polat and Tokgöz, 2014). A content analysis of the Twitter messages among Turkish users was performed to make meaning of those messages. Another study examined up to nine months of Twitter interactions to discover how this social media news platform was being used in the top ten Canadian HE institutions to brand and market themselves online (Veletsianos et al. 2017). Further, how did these social media interactions affect faculty and student life? Veletsianos et al. (2017) summarized their findings into four characteristics of institutional social media accounts. Each social media account messages served as a narrative using social construction of technology theory to explain for the overall branding and marketing strategies incorporated in the administering of each HE institution online. In short, these studies were conducted solely based on the authors' research interests to see how Twitter communications are a part of professional development, organizational leadership, and teaching and learning. Another recent study (Peacock et al. 2016) provides an exemplary survey based on a 5-point Likert scale that provides insights from a teaching staff's perception of their espousal of Twitter usage as it pertained to a popular culture project at a mid-sized southeastern university in the US. Unlike former studies, the main research goal was neither to dissect nor analyze the content of the Twitter messages for the intent of making sense of it. This study, therefore, is a significant contribution towards examining the different online leadership constructs in higher education across those of different countries, namely the US, UK, Canada, and the Republic of Korea. An original contribution to the existing literature on DL is illustrated with the practical extension of knowledge management in the digital confines of HE. It is the first research to propose DL online by quantifying the twenty-one leadership activities and making 
sense of the public communications, namely, tweets, and how DL in practice is reconfigured digitally in nuanced ways, for which I explained above, as well for strengthening how it is viewed synonymously to the notion of memos, implied by Spillane.

\section{III- METHODOLOGY}

\section{Research Design}

The impact of leadership that is conducive to different online cultures across the US, UK, Canada, and the Republic of Korea can be addressed by the twenty-one administrative activities of online leadership, which I had created. Learning opportunities and outcomes are to be considered for each administrative discussion that is initiated online. Such activity-related constructs will serve as binary variables for each post on Twitter (Gough, 2017b).

Figure 1. Twenty-one Leadership Activities

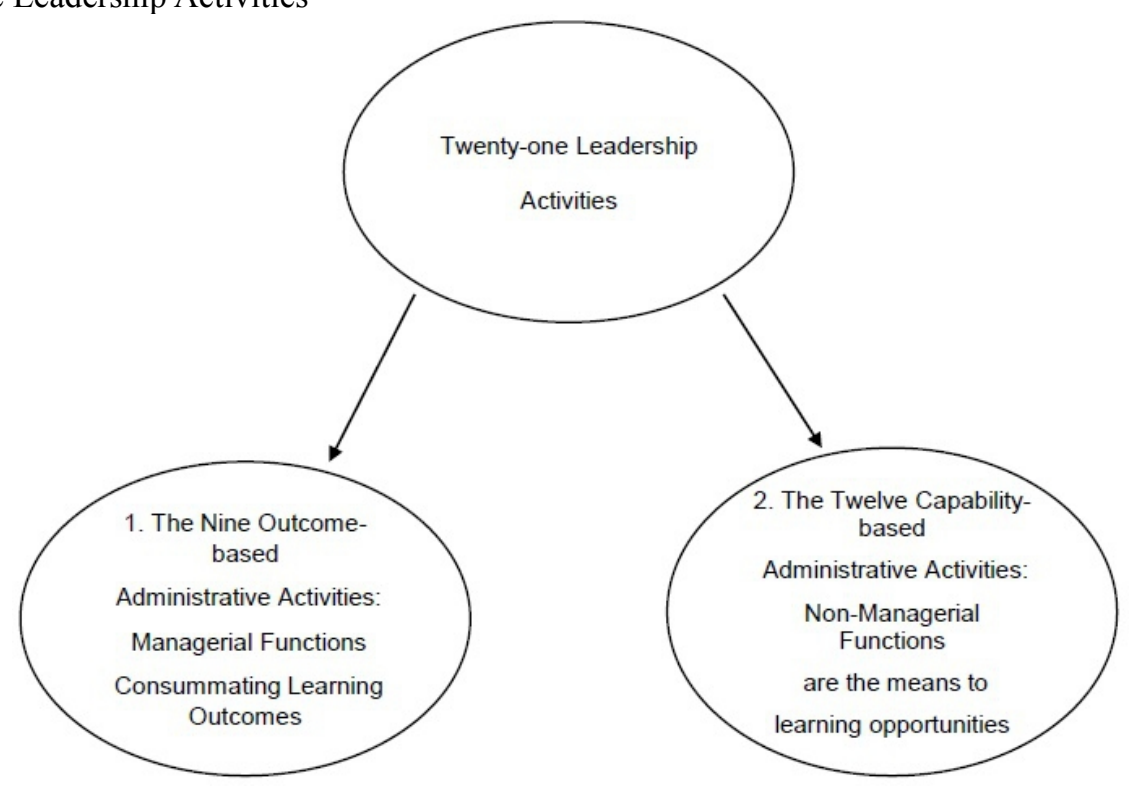


After all the posts' contents have been parsed for the appropriate managerial and nonmanagerial categories of $\mathrm{HE}$ activities, the values will be aggregated into frequency tables for each activity to see the impact of each indicator; and lastly, there is no double counting for each entry falling into either a managerial or non-managerial activity.

Previous research on six corporate businesses in the US and Australia illustrates through the models of interactive communications in consumer marketing that are applicable in the Twitterinteraction context (Burton and Soboleva, 2011). Sears (2015) explains that Twitter's creativity content channels can be exploited towards strengthening personal and corporate leadership, if strategically used. These research studies provide the impetus for creating the nine different types of managerial functions involved in the intimate Twitter communications being conveyed by both the administrative leadership and its users. These nine categories of leadership activities, representing the learning outcomes to be considered for contextual analysis, and to derive statistics from, are the following (as proposed in Figure 2): HE governance, staff-centered administrative leadership, educational leadership, student-run government leadership, administrative guidance leadership, community editorial leadership, active community development (activities and events), socio-economic inequality and cultural discussions, and finally, STEAM-related leadership. 
Figure 2. The Nine Outcome-based Administrative Activities

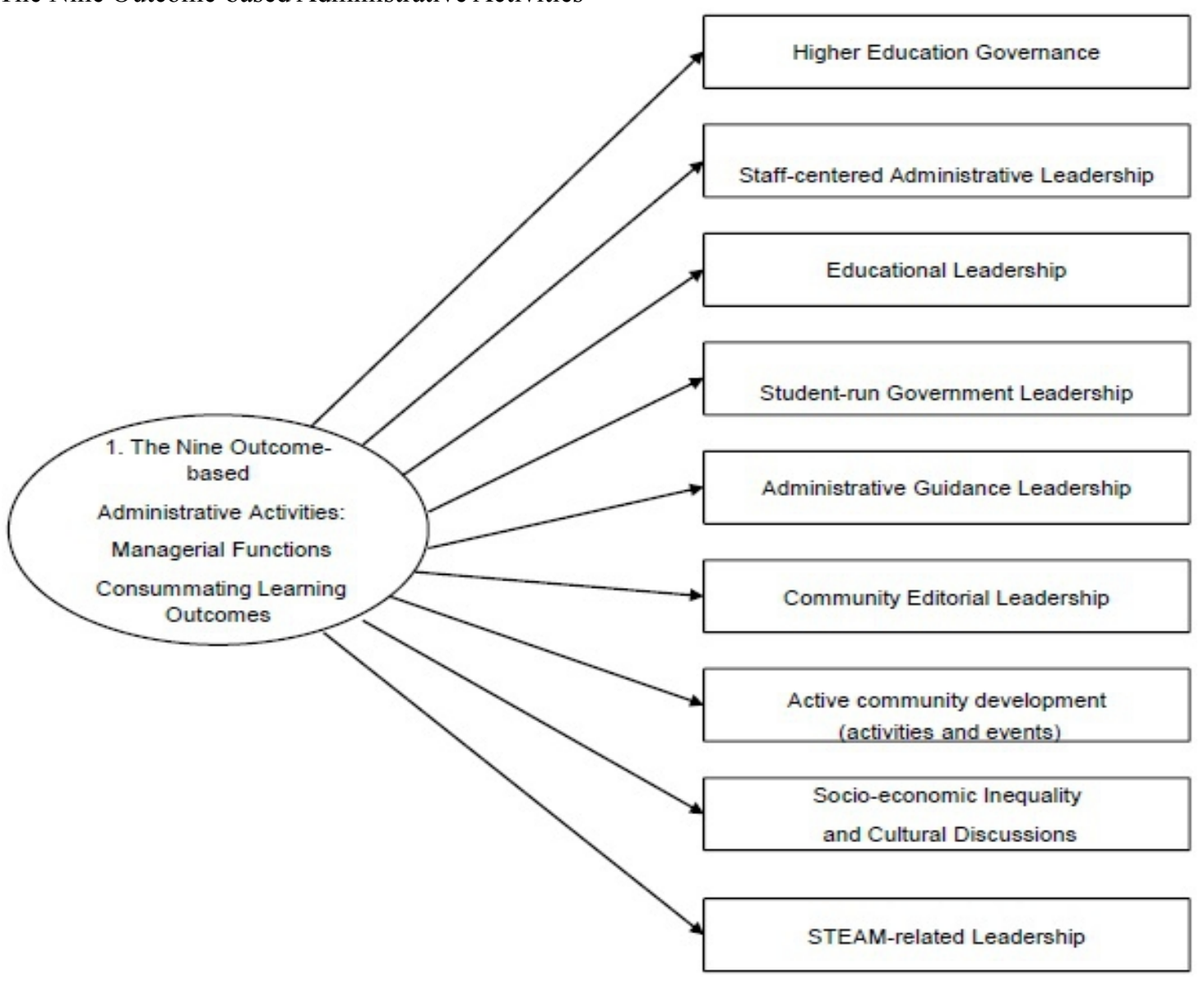

Higher education governance leadership involves the power to authorize any relevant decisionmaking and policy-making with respect to hierarchical structures, such as faculty, academic committees, senates, and boards, in addition to the management of alumni affairs. Administrative structures are not just confined to the likes of departments, and programs, but also includes executive administration, such as the Office of the President, Provost and Chancellor. Together, the university as whole, or independently, a specific department, the institutional division may operate and function as a connected entity to the broader network of universities and institutions. Staff-centered administrative leadership includes mission and goals, positions, grants, rewards, scholarships, proposals, 
forum/debate, promotions, hiring, announcement of disclosure of annual report and financial statements, new course offerings, someone's research published, talented foreign leaders and speakers being invited, and windfalls in professor-student research to list some of the descriptors. Next, educational leadership involves pedagogy, standards, curriculum, trends, and school improvement. Student-run government leadership is a role promoted by any entity that organizes on behalf of the the student government. Administrative guidance as a leadership role is the support system addressed for directing academic success. Community editorial leadership includes opinions, state-wide education legislature, presidential elections, and presidential leadership. Leadership through community development activity programs may seek community members to help strengthen relations among them. Leadership concerning socio-economic inequalities and culture has to do with leading the discussion for improving the state of the social, economic, and cultural milieu. Lastly, STEAM-related leadership can be about the sciences, Maker Movements, 3D printing technologies, coding and programming, and mathematics in relation to aesthetics or the arts (see table 8, in the Appendix, to see a sample of the actual texts being broadcast under each category, as there are nine outcome-based managerial functions denoting leadership activity trajectories).

The other twelve non-managerial, capability-based activities follow as learning opportunities (as proposed in Figure 3) delved into events for students, events for the community (friends and family), research, available services on campus, about students, staff, and communities, about local news (other departments, neighbourhood, community, province, region), national news, international news, knowledge and resource sharing, inspiration and motivation, technical information, and benefits to learning. Table 9 in the appendix provides specific examples for each of the twelve possible nonmanagerial capabilities to learning. 
Figure 3. The Twelve Capability-based Administrative Activities

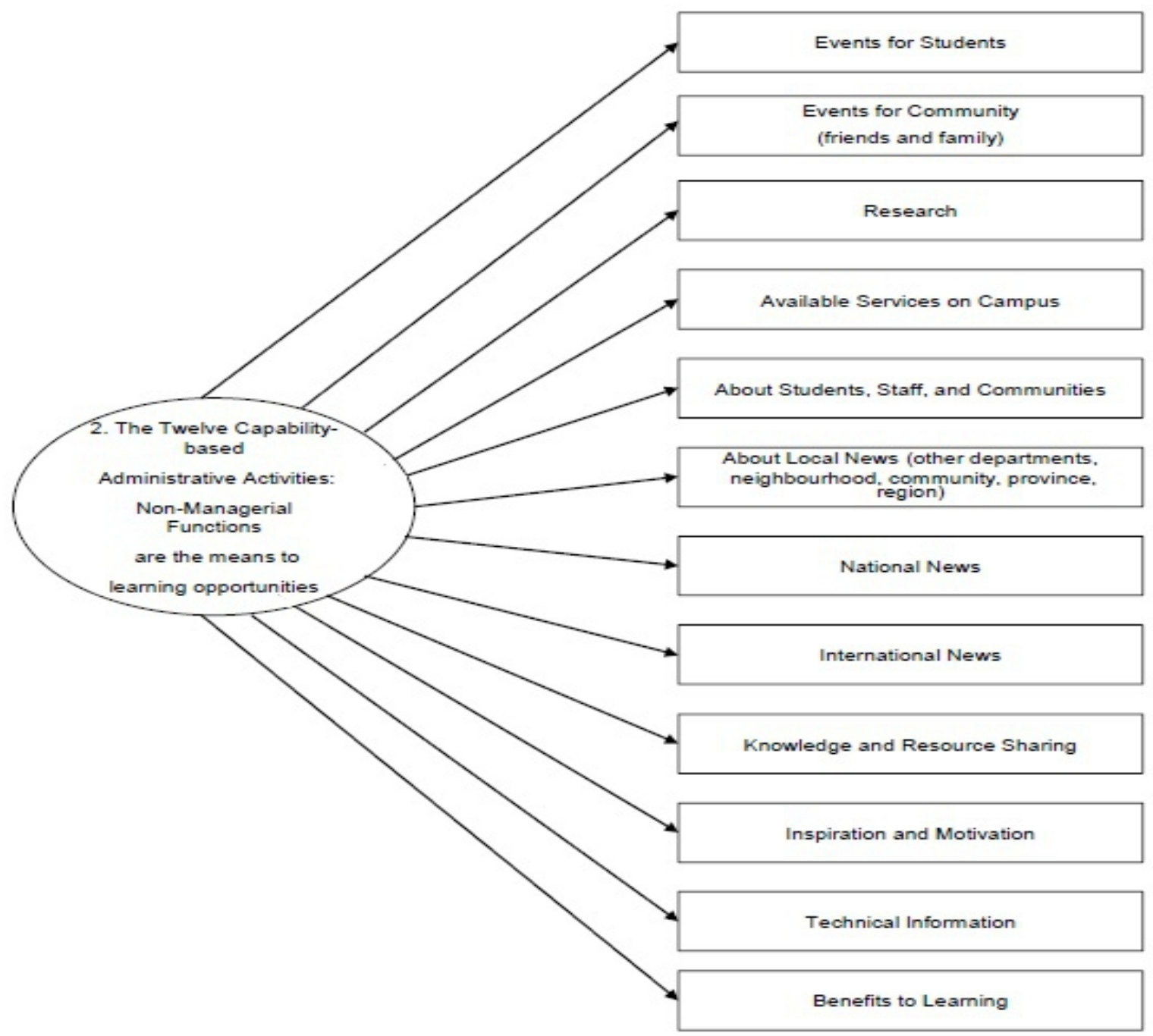

\section{Higher Education Institutions as Participants}

Stanford University was a perfect candidate because of the plethora of Twitter profile pages of each faculty, and sometimes, programs and research teams broadcasted news to fit their purpose and audience it wished to attract. The Faculty of Education's (@StanfordEd) Twitter page was analyzed. 
University of Alberta, a Canadian university was selected due its top placement in the Google search engine result when searched for a Canadian HE Twitter profile. For England, Cambridge University appeared as the most searched HE in the UK. Although its (@CambridgeEdFac) Twitter page is much similar to the Korean universities with low interactions to their communications, it provided a niche where the organizational culture flourished over time. Because there was inadequate data to compare against the robust information available on @Education_UofA (University of Alberta's Education department) and@StanfordEd Twitter profile pages, I searched for a popular Twitter account in England, and discovered University of Oxford Continuing Education (@OxfordConted), that amplified its leadership online. Unfortunately for Korea, the Departments of Education do not own, or have managed Twitter, although accounts exist at the university-wide level.

Focusing on Korea's take on online leadership via Twitter, seven South Korean universities considered are as follows: Seoul National University, Korea University, Yonsei University, KAIST, Cyber University of Korea, Seoul Cyber University, and Ewha Womans University. However, Cyber University of Korea and Seoul Cyber University did not have Twitter profile pages, so they were exempted from the analysis of this study. It is ironic that these top online universities in South Korea do not have Twitter accounts when traditional offline South Korean universities have extended their business and education beyond the physical walls of the classroom into cyberspace. It would be interesting for future research to take a look at how the cyber universities use similar online platforms to connect with their student body at large. As a result, five South Korean universities were analyzed for functional-usage statistics and contextual meanings to be decomposed into leadership styles. Figure 4 provides a live example of Ewha Womans University's Twitter page (Gough, 2017a). 


\section{Data Collection}

The first sample of observations, $n=201$ Tweets, for the chosen Western HE institutions in a given month is specifically from June 2016 through August 2016. This month-long time frame is necessary and sufficient to produce valid data that includes an ample demonstration of online leadership; moreover, I constructed the nine managerial functions and the twelve non-managerial opportunities of leadership, that are addressed in a public manner in an online community, via Twitter. Compared to previously noted studies, this analysis expands the window to a full month, as the robust data provides insights that other online leadership articles did not explore.

The second sample of observations, $n=297$ tweets, comes from the chosen South Korean HE institutions in a given year that ranges from July 2015 through July 2017. As for Seoul National University and Korea University, data is collected from July 2015 through July 2016, while for Korea Advanced Institute of Science \& Technology (KAIST) and Ewha Womans University, data covers the entire, current year. This year-long period makes it sufficient for the data to validate a reasonable demonstration of online leadership that can be categorized into the twenty-one leadership activity roles, consisting of either the nine possible outcome-based, managerial functions, or the twelve possible capability-based, non-managerial opportunities towards teaching and learning (as I propose in Figure $1)$.

The third sample of observations consisted of a manual scan of 14,183 tweets was performed from the selected education department or university from its Twitter profile activation date through August 31st, 2017. Again, overall frequencies are taken. 


\section{Analytical Procedure}

The meat and butter for the analytical procedure are derived from what Twitter provides in terms of its three basic functions for each independent post: the 'tweet' (actual content) itself, 'retweet' (repeated posts of third party content), and to 'like' options. These three automated activities that simultaneously syndicate in real-time virtually to everyone else in the network provides the Twitter account holder and its audience the power to observe and monitor all the frequencies of replies, retweets, and likes for each conversation with text being limited to a 140-character-length statement. Based on these numbers, we can provide frequencies (and possibly, descriptive statistics) to further analyze these microblogs into a context of educational technology, interfacing with online leadership as the practical strategy towards improvement and effectiveness in the US, UK, Canada, and South Korea.

\section{Specific Scope of the Study}

Success of online DL will hinge on the trustworthiness and the willingness of people to react with clear emotional signals and insights. Long-term conversations and experience of others will further meet desirable strategic leadership goals. The dissemination of inspiration thus repeats itself through the categories manifested in the capabilities and outcomes. Knot-working (Engeström et al. 1999) is similar to how the online participants on Twitter respond and voluntarily work with the Higher Education Institutions (HEIs) in terms of their likes, retweets, and direct replies. Differences in West and East towards DL styles purveyed by Twitter can also be understood as cultural knot-working, which is a spontaneous collaborative embodiment that matches Gronn's concerted leadership style (2002). 


\section{IV- FINDINGS}

\section{Context}

The designation of both managerial functions and non-managerial administrative activities as indicators of learning outcomes and opportunities, respectively, in a frequency analysis framework is a good means to measure the content and purpose of the online Twitter activities expressed by each HE institution since the three research questions will be answered in details. I am assuming that the sender of each institution's tweet is syndicated by the authorized public relations (PR) team, and that because the PR team takes suggestions from others in the community to post each tweet, DL is enhanced rather than inhibited. However, a simple experiment can be conducted with the likes of van Ameijde et al (2009) to allow key university representatives and administrators to hold a separate Twitter account, or

by having the PR reveal identities, whose contributions wound up as ideas or sources for the tweets, if each administration decides to keep a single university or sole department account. Deeper investigation can scientifically detect whether the various senders in different levels of authority and administrative functions would enhance or inhibit DL that is concerned with the precision in leadership handling.

\section{Emotional Responses}

This primary research question treats how frequently each Twitter post, known as Tweets, including photos and videos, is distributed by the university or education department as an activity that allows its followers, connected in the network, to internalize the situated process, such that all individuals' expressed emotions and thoughts are observed in terms of frequencies that may pinpoint trends in responses to dynamic leadership approaches. These emotional responses to the Tweets are 
aggregated as frequencies and relative frequencies to compare the meaningful accumulation among academic institutions for all interactions within the organizational design, such as the frequency of 'likes' during the recent time periods. Firstly, the frequency information collected (in Table 1, below) pinpoints, by country, the frequencies of Twitter posts launched by the education department or university spanning from the activation date on Twitter to the present day, August 31st, 2017, along with the number of followers and the frequencies of the followers' liking each activated Tweet. The frequency of 'likes' supports the emotional responses given by users in the online community for every administrative activity. By country comparisons, the US has the most frequent number of Tweeted activities standing at 6,321 posts (and a relative frequency of $44.57 \%$ ) with the second most amount of photos and videos presented as a relative frequency. Canada is second place in terms of frequencies for its Tweets and the amount of followers, but it is actually first place for the members on Twitter it is actively following (75.66\%), the most amount of likes it received (relative frequency of $65.24 \%$ ), and for having the most photos and videos linked in the posted messages (relative frequency of $37.38 \%$ ). The number of followers to South Korean academic institutions is lowest at 5004 (or 12.85\%), with an expectant relative frequency of being 'liked' at $1.53 \%$. The UK's frequency of Tweets and the interaction by its followers are slightly better than South Korea's position.

In summary of the findings for each country by comparison, the frequency of Tweets coincides with the number of followers, yet it seems that the more an institution pursues its followers by opting to follow others as opposed to having members following it, the result is more of its followers liking its activities. Also, if the more the Tweets contained images and videos, the more likelihood there is for people willing to follow or appreciate their activities. Reverting to DL Theory, the Tweets are stretched across its followers as a whole activity, yet the institution decides who it will follow, as its members of 
the online community may collectively interact and influence the perspective being shaped for its administrators, faculty, and student body. Western faculty departments of education are keen on frequently distributing its activities to be cast on the followers' desire to engage with leadership roles in an institutional setting that are contemporary, practical, dynamic, and sociable. As for the S. Korean narratives, each institution demonstrates that the distributed perspective is not completely lacking in the network approach to engage its emergent leaders. Interactions to and from the administrative activities by S. Korean universities convey its nascent, baby steps in the path to following the techniques of organizational success during the current industrial wave (Gough, 2017b; Eom, 2017).

Table 1. Frequency of Tweets and Interactivity by Country for the Nine Academic institutions on Twitter, since activation date to the present (August 31st, 2017)

\begin{tabular}{|l|c|c|c|c|c|c|c|c|c|c|}
\hline \multicolumn{2}{|l|}{ Summary of Twitter Interactions for Academic Institutions } \\
\hline Country & $\begin{array}{c}\text { Tweets } \\
\text { (f) }\end{array}$ & $\begin{array}{c}\text { Tweets } \\
\text { (rf) }\end{array}$ & $\begin{array}{c}\text { Number } \\
\text { of Twitter } \\
\text { Accounts } \\
\text { following }\end{array}$ & $\begin{array}{c}\text { Percentage } \\
\text { of Total } \\
\text { Twitter } \\
\text { Accounts } \\
\text { Following } \\
(\%)\end{array}$ & $\begin{array}{c}\text { Number } \\
\text { of } \\
\text { Followers }\end{array}$ & $\begin{array}{c}\text { Percentage } \\
\text { of Total } \\
\text { Followers } \\
\text { (\%) }\end{array}$ & $\begin{array}{c}\text { Likes } \\
\text { (f) }\end{array}$ & $\begin{array}{c}\text { Likes } \\
\text { (rf) }\end{array}$ & $\begin{array}{c}\text { Photos } \\
\text { and } \\
\text { Videos } \\
\text { (f) }\end{array}$ & $\begin{array}{c}\text { Photos } \\
\text { and } \\
\text { Videos } \\
\text { (rf) }\end{array}$ \\
\hline $\begin{array}{l}\text { United } \\
\text { States }\end{array}$ & 6321 & $44.57 \%$ & 129 & $2.63 \%$ & 22100 & $55.86 \%$ & 321 & $9.08 \%$ & 774 & $34.90 \%$ \\
\hline $\begin{array}{l}\text { United } \\
\text { Kingdom }\end{array}$ & 2089 & $14.73 \%$ & 447 & $9.12 \%$ & 5989 & $15.14 \%$ & 854 & $24.15 \%$ & 615 & $27.73 \%$ \\
\hline Canada & 4209 & $29.68 \%$ & 3709 & $75.66 \%$ & 6470 & $16.35 \%$ & 2307 & $65.24 \%$ & 829 & $37.38 \%$ \\
\hline $\begin{array}{l}\text { South } \\
\text { Korea }\end{array}$ & 1564 & $11.03 \%$ & 617 & $12.59 \%$ & 5004 & $12.65 \%$ & 54 & $1.53 \%$ & NA & NA \\
\hline
\end{tabular}

\section{The 9 Outcome-based Administrative Activities (Managerial Functions)}

The frequencies of nine outcome-based administrative activities were addressed in the second research question. The more frequently those managerial functions are distributed across the social 
network, the more learning outcomes can be consummated. In the managerial context of the distributed perspective, outcome-based activities contain varying degrees of administrative trajectories towards learning opportunities, and the tendencies of such defined roles are measured. Thus frequencies of activities within the management context convey a stronger devotion towards the administration of staff and the development of its community, while Stanford exudes confidence towards the leadership within socio-economic culture and STEAM-related research pursuits. Oxford distinctly achieves managerial concentration in its faculty, hiring and staff-related operations with a slight foregrounding of managerial enlightenment to HE governance and STEAM-related matters. University of Alberta's is also dynamically covering most of the learning outcomes, particularly pursuant in the management of HE governance and community editorials.

In addition, the second research question appeals to frequencies of managerial functions that characterize the leadership styles for teaching and learning. Ewha is most enthusiastic about its managerial efforts in that 7 of its 8 ongoing outcome-based activity functions merit the highest likelihood of commitment in administrative guidance. With the frequencies alone, Ewha appears to be the most open to gain from reorganizing its leadership structure and style to fit into the new challenges and demands of its students and faculty members. KAIST follows with managerial strengths after Ewha in that it positions well with STEAM-related development, faculty and staff hiring, and with promoting matters. Both Korea University and SNU achieve a balanced touch to six ongoing managerial outcomes; and KU is concerned with educational leadership and governance issues. Compared to the previous year, the dynamic and persistent managerial roles in the online context are living up to the world-class nature of these universities, except for Yonsei University that is absent in these efforts, only Ewha has proven to be most eager to express DL via online leadership to its faculty, 
student, and general followers.

DL is still tangent with the leadership activities and functions that are encouraged among different individuals to align with its participatory culture within the organization (Spillane et al. 2004), which contribute to its organizational culture of shared collaboration and responsibilities (Hargreaves and Fink 2008; Spillane et al. 2007a.; Storey, 2004). DL theory has been conducted and applied more pervasively in the UK than in the US, in terms of manifesting its administrative co-ordination of employee relations towards school improvement (Bolden, 2011; Goleman, 2002). In the case of S. Korea, there was a lack of communication in personal stories, or narratives of students, faculty, and staff being shared online. Thus, there is a sense of separation between the people directly involved in HE and S. Koreans. A clear sense of the traditional, sole leader approach is manifested in these Tweets promulgated in S. Korean HE. To apply DL, restructuring of the organization (Hargreaves, 2007; Spillane, 2006) is required to induce organizational culture that fits the changes for necessary improvements.

\section{The 12 Capability-based Administrative Activities (Non-Managerial Opportunities)}

The third research question, therefore, involves the frequencies of non-managerial functions as administrative capabilities, which are distributed across the social network as learning opportunities. For both Western and Korean academic institutions, higher tendencies of capabilities being enacted results in a positive increase in the exchange of conversations in educational matters, such as event planning, research conduct, available services on campus, about faculty and students, news citations, new knowledge and resource-sharing, inspiration and motivation, technical information, and benefits to learning. 
In the non-managerial context of the distributed perspective, capability-based activities contain varying degrees of administrative trajectories towards learning opportunities, the tendencies of such defined roles are quantified. In comparison, @Education_UofA makes more tweets, usually tweets 5 messages, while @StanfordEd tweets less with about 3 messages daily based on the one month study. However,@StanfordEd is far more active in terms of participation from the audience with higher volumes of likes and replies - something that is lacking in the quantity of the tweets being broadcast by the UK and South Korean HE institutions. Knowledge and resource-sharing with event planning and a slew of other high multiple capabilities as learning opportunities is the hallmark of University of Alberta Faculty of Education, whereas Oxford University Department for Continuing Education is generally a low-capability performer. As per observations in the previous year's month (refer to Table 7), Ewha can be commended for having more of an administrative role being distributed across the social network in the non-managerial context compared to Cambridge University Faculty of Education (in Table 5), especially when non-managerial functions for the other S. Korean universities are mostly absent. KAIST's leadership style is keen on STEAM-related administrative functions and learning outcomes, in addition to the capability-based technical information, but its impetus for teaching and learning as an administrative opportunity within community events is well-rounded with a strong finish in international news, coupled by its power in sharing of its knowledge base and resources.

Favorable circumstances for learning are situated as encouraging opportunities towards the greater interests of its social network users. As it becomes frequent for its users to respond to the distributed capabilities in the greater leadership perspective, the more administrators will know in dealing with the public's iterative "responses to responses" that are common to a growing web of interactions. Flow of information passes to the designated leaders from the following crowd, forming 
potential critical mass to a situational awareness of the needs, wants, and what the people actually think and know. These techniques in organizational skills allow all users to become experts. Creativity is also enhanced as demands and changes in the social environment are perceived as fond challenges to solve new problems and tasks. So, the more frequently the social network knows its people, the more opportunities in learning will become available.

\section{V-Discussion, Conclusion and Recommendations}

\section{Discussion}

The observations on Twitter will eventually amount to big data, and the methodology presented in this research gains strength from each proceeding online post to the accumulation of various sentiments and feelings through which fruitful interactions bring. Hence, as leadership activities are being distributed across the social network in a collaboration process, online leadership shall improve over time for being a product of interactions by its followers, and so forth. The administration that is behind the online presence can stimulate the distributed process. An analytical mindset takes the social network approach with respect to a better understanding of people's goals to reach new beginnings, a brighter future, and success. Thus opportunities in learning are a commitment of non-managerial administrative functions. Taking on such leadership role and responsibilities relies on analysis to forecast the effects of their decisions and plans.

As probed by the first research question, the distributed frequency of emotional responses is a reflection of the Tweets being dynamic and practical about how leadership as a distributed perspective is improving the state of teaching and learning, in addition to remain renewed, relevant, aspiring, 
intellectual, diplomatic, progressive, interesting and innovative. There is agreeance with the statement that the collective online output is greater than the sum of their individual assertions. That stated, the broader implication of DL is comprised of its openness to widen its stretch to a fuller network of participants.

The frequencies of nine outcome-based administrative activities were addressed in the second research question, and the more frequently those managerial functions are distributed across the social network, the more learning outcomes can be consummated.

Perhaps, a social network analysis approach addresses how leaders, whether independent or group-minded, are developed and formed within a reorganization of conventions (Fransen et al. 2015; Mehra et al. 2006; Balkundi and Kilduff, 2006). In addition, the nine outcome-based, functional leadership activities also spur the roles that individual university students and other members of the network may undertake to enrich the distributed perspective on managerial tasks and learning outcomes.

Both the institutions in the West and S. Korea frequently furnish outcome-based HE governance and staff-centered administrative leadership roles, but Ewha Womans University and KAIST are managerial trailblazers for certain learning outcomes compared to Oxford University and University of Cambridge. Stanford and University of Alberta provide distinct leadership styles that steer others to them that results in more emotional responses, as discussed in the first research question.

Achieving such an order through the process of restructuring in the case of social media usage can also be cumbersome, as this is highlighted in South Korea's adoption of online leadership in the HE sector. At any rate, however, there may be a tipping point in the near future in South Korea as there is strong advocacy towards the Fourth Industrial Revolution. Although the decision-making is made 
accessible across the institution and leadership tasks are considered a shared responsibility among its individuals depending on the situation (Spillane et al. 2007a), no research is being carried out to study the DL practice that is immersed in Twitter, particularly in HE. A future study can provide more details on how decision-making is done on Twitter.

As the third research question showed, South Korean HE institutions, however, have less competency maneuvering and administering activities concerning events intended for students and community, local news, as well as the offering of inspiration and motivation compared to its Western counterpart. Regardless, both the Western and S. Korean institutions have the highest frequencies for its capabilities in sharing knowledge and resources, which is one of the top learning activities, followed by events for students and community. As the Korean universities are becoming more open with the idea of DL, the yearly data hosts substantive criteria, whereas monthly data would rather be uncertain for the time-being. Ewha Womans University is exceptional in sharing the nine outcome-based leadership roles as much as it has relayed its 12 capabilities for administrating the learning opportunities as its means. Hence, Ewha's leadership style has a strong balance in the leadership role with respect to the twenty-one possible activities as a whole.

Twitter that is being used by the Korean HE is an exemplary extension of the traditional reality, where the organizational boundary is rigid. As such, Korean HE has the salient characteristics embedded in the societal differentiation and rationalization, as well as specialization in terms of hierarchy, division of labor (talent) and managerial control (Gordon, 2002).

In the traditional sense, there are clear limitations to power with defined boundaries of hierarchy and rational authority. In the West, however, the department of Education provides a testimony to the flexible, yet traditional organizational boundary, due to a collaborative organization and the sharing of 
the resources and information (Gordon, 2002). Therefore, the exchange of information and collaboration on Twitter can be treated as a brokerage for power. As in the previous discourse on the possibilities exchanging memos online, namely through the alternating roles of leader, follower, and observer in the exchange of e-memos, tweeting has done justice to promote a balance of power amongst those taking initiative in the network. This conceptual undertaking not only consummates DL as a theory working in practice, but also manifests the barriers that are eliminated with online leadership.

\section{Conclusion}

After reading a total of 14,183 Tweets and deeply analyzing 498 Tweets collected from five universities and four education departments in four different nations, each resultant social network had a clear online persona and a distinct leadership style. This study is the first to measure and predict the opportunities and outcomes backed by the predisposed capable and functional leadership trajectories for teaching and learning activities. Leadership styles are dictated by the pattern of tasks and roles being distributed across the followers in the network over instantaneous time on Twitter. DL theory is rather influenced by the possibilities being materialized by the online leadership of HE institutions.

The direct contextual analysis of Twitter usage patterns and content demonstrates how collegial aspects that previous research deemed to be lacking due to "new managerism" are in fact important. This new form of education augurs well with the purpose of moderating DL. Similarly, van Ameijde et al (2009) state that the new managerism in HE can promote DL to better maintain collegial aspect and professional autonomy. This study successfully utilized Twitter as the online platform to apply DL Theory. 
Reverting to the online leadership styles being practiced in different universities, preferences for the authoratative structure that exists online between HEI and its members can be traced to the 21 capabilities and learning outcomes. Moreover, Twitter has been exploited in ways to empower lowerlevel employees with broader decision-making responsibilities, and thus, a spill-over effect of the administrative power towards the lower levels of status in the organization. The alternating voice for authority and management in HE, as well as responsibilities taken for either leading and following amongst its members, are thus spread across beyond the grid of distributing knowledge in the learning opportunities and outcomes.

The West is a good example for empowering their followers, yet differentiating themselves without imposing dominance and instances of an imminent power struggle to the followers. Adding to the theory, the approach to discern the exercise of leading by Tweeting, and so forth, by separating the administrative activities as capabilities and learning outcomes, a new conceptual understanding about the strengths, limitations, possibilities, and uncertainties can be made. While also contributing to DL in practice, this particular approach to DL clearly demonstrates that anyone with access to the tweets being cast by the HEIs may freely exercise their right to perform distributed leadership.

\section{Recommendations}

I recommend that practitioners, such as administrative information and technology managers to explore the many options to undertake the optimal distributed perspective. As people begin to rely on the online leadership, more users will trust the intelligence of others with individual motivation, drive,

and independence in tact over social media connections. Such non-managerial aspects of leadership that are enacted online are the means to increase the speed and quality of cognition affected by the 
potential intensity of the emotional connections.

Future research can further add to this study's significant findings on the DL in the online leadership context where such practical plans can be implemented in the contemporary cultural contexts and geographic boundaries that differ.

\section{REFERENCES}

Balkundi, P., \& Kilduff, M. (2006). The ties that lead a social network approach to leadership. The Leadership Quarterly, 17, 419-439

Blaschke, S., Frost, J., \& Hattke, F. (2014). Towards a micro foundation of leadership, governance, and management in universities. Higher Education, 68(5), 711-732.

Bolden, R. (2011). Distributed leadership in organizations: A review of theory and research. International Journal of Management Reviews, 13(3), 251-269.

Burton, S., \& Soboleva, A. (2011). Interactive or reactive? marketing with twitter. The Journal of Consumer Marketing, 28(7), 491-499.

Cho, V. (2016). Administrators' professional learning via twitter. Journal of Educational Administration, 54(3), 340-356.

Engeström, Y. (1999). Innovative learning in work teams: Analysing cycles of knowledge creation in practice, in: Y. Engeström et al (Eds.) Perspectives on Activity Theory, (Cambridge, Cambridge University Press), 377-406.

Eom, S. (2017, June). 제4 차 산업혁명시대의 대통령 리더십-강사세계의 실제세계의 역동을 중심으 로 Paper presented at the 제 178 회 국가리더십 포럼, 서울대학교 행정대학원 57 동 204 호

Floyd, A., and Preston, D. (2017). The role of the associate dean in UK universities: distributed leadership in action? Higher Education, 75, 925-943. DOI 10.1007/s10734-017-0178-1

Fransen, K., Van Puyenbroeck, S., Loughead, T., Vanbeselaere, N., De Cuyper, B., Vande Broek, G., \& Boen, F. (2015). Who takes the lead? Social network analysis as a pioneering tool to investigate shared leadership within sports teams. Social Networks, 43, 28-38.

Goldstein, J. (2004). Making sense of distributed leadership: The case of peer assistance and review. Educational Evaluation and Policy Analysis, 26, 173-197. 
Goleman, D. (2002). The new leaders: Transforming the art of leadership into the science of results. London: Little Brown.

Gordon, R.D. (2002). Conceptualizing leadership with respect to its historical contextual antecedents to power. Leadership Quarterly, 13, pp. 151-167.

Gough, S. (May, 2017a). Online leadership of higher education for the departments of education in the US, UK, Canada, and South Korea. Unpublished essay.

Gough, S. (August, 2017b). Online leadership of higher education for the departments of education in the US, Canada, UK, and South Korea. Unpublished manuscript.

Gronn, P. (2008). The future of distributed leadership. Journal of Educational Administration, 46(2), 141-158.

Gunter, H. M., Hall, D., \& Bragg, J. (2013). Distributed leadership: A study in knowledge production. Educational Management Administration \& Leadership, 41(5), 555-580.

Hargreaves, A. (2007). Sustainable leadership and development in education: Creating the future, conserving the past. European Journal of Education, 42(2), 223-233.

Hargreaves, A., \& Fink, D. (2008). Distributed leadership: Democracy or delivery? Journal of Educational Administration, 46, 229-240.

Harris, A. (2004). Distributed leadership and school improvement: Leading or misleading? Educational Management Administration \& Leadership, 32(1), 11-24.

Harris, A. (2005). Crossing boundaries and breaking barriers: Distributed leadership in schools. London, UK: International Network for Educational Transformation.

Harris, A., \& Spillane, J. (2008). Distributed leadership through the looking glass. Management in Education, 22(1), 31-34.

Leithwood, K., Mascall, B., Strauss, T., Sacks, R., Memon, N., \& Yashkina, A. (2009). Distributing leadership to make schools smarter: Taking the ego out of the system. Leadership and Policy in Schools, 6(1), 37-67.

Mehra, A., Smith, B., Dixon, A, \& Robertson, B. (2006). Distributed leadership in teams: The network of leadership perceptions and team performance. The Leadership Quarterly, 17, 232-245.

Peacock, J., Covino, R., Auchter, J., Boyd, J., Klug, H., Laing, C., \& Irvin, L. (2016). University faculty perceptions and utilization of popular culture in the classroom. Studies in Higher 
Education, DOI:10.1080/03075079.2016.1180673

Polat, B., \& Tokgöz, C. (2014). Twitter user behaviors in Turkey: A content analysis on Turkish Twitter users. Mediterranean Journal of Social Sciences, 5(22), 244-251.

Sears, S. M. (2015). How to play-and fix-twitter. Barron's, 95(27), n.p.

Spillane, J. P., Camburn, E., \& Pareja, A. S. (2007). Taking a distributed perspective to the school principal's workday. Leadership and Policy in Schools, 6, 103-125.

Spillane, J. P., Camburn, E., Pustejovsky, J., Pareja, A. S., \& Lewis, G. (2008). Taking a distributed perspective: Epistemological and methodological tradeoffs in operationalizing the leader-plus aspect. Journal of Educational Administration, 46(2), 189-213.

Spillane, J.P., Halverson, R., \& Diamond, J.B. (2004). Towards a theory of leadership practice: A distributed perspective, Journal of Curriculum Studies, 36(1), 3-34.

Spillane, J. P., Halverson, R., \& Diamond, J. B. (2007). Investigating school leadership practice: A distributed perspective. Educational Researcher, 30, 23-28.

Spillane, J.P. (2006). Distributed Leadership. San Francisco: Jossey-Bass.

Stensaker, B., \& Norgård, J. D. (2001). Innovation and isomorphism: A case-study of university identity struggle 1969-1999. Higher Education, 42(4), 473-492.

Storey, A. (2004). The problem of distributed leadership in schools. School leadership and management, 24(3), 249-265.

Twitter. (2017). Welcome to Twitter. Homepage. www.twitter.com. Accessed on 16 June 2017.

Van Ameijde, J. D. J., Nelson, P. C., Billsberry, J., \& van Meurs, N. (2009). Improving leadership in higher education institutions: A distributed perspective. Higher Education, 58(6), 763-779.

Veletsianos, G., Kimmons, R., Shaw, A., Pasquini, L., \& Woodward, S. (2017). Selective openness, branding, broadcasting, and promotion: Twitter use in Canada's public universities. Educational Media International, 54(1), 1-19. 